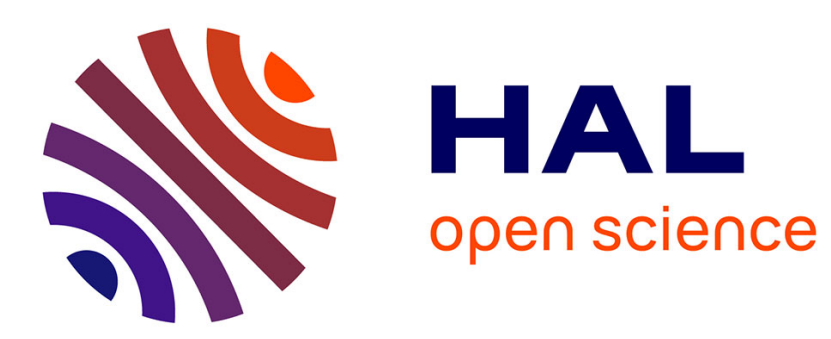

\title{
Relations conductivité/microstructure dans des céramiques composites superconducteur (NASICON)/verre isolant
}

Ph. Colomban

\section{- To cite this version:}

$\mathrm{Ph}$. Colomban. Relations conductivité/microstructure dans des céramiques composites superconducteur (NASICON)/verre isolant. Revue de Physique Appliquée, 1987, 22 (8), pp.719-727. 10.1051/rphysap:01987002208071900 . jpa-00245601

\section{HAL Id: jpa-00245601 https://hal.science/jpa-00245601}

Submitted on 1 Jan 1987

HAL is a multi-disciplinary open access archive for the deposit and dissemination of scientific research documents, whether they are published or not. The documents may come from teaching and research institutions in France or abroad, or from public or private research centers.
L'archive ouverte pluridisciplinaire HAL, est destinée au dépôt et à la diffusion de documents scientifiques de niveau recherche, publiés ou non, émanant des établissements d'enseignement et de recherche français ou étrangers, des laboratoires publics ou privés. 
Classification

Physics Abstracts

$66.30 \mathrm{H}-78.30-81.20 \mathrm{Q}$

\title{
Relations conductivité/microstructure dans des céramiques composites superconducteur (NASICON)/verre isolant
}

\author{
Ph. Colomban \\ Groupe de Chimie du Solide, Laboratoire de Physique de la Matière Condensée, \\ G.R. 38 du CNRS, Ecole Polytechnique, 91128 Palaiseau, France
}

(Reçu le 5 mars 1987, accepté le 28 avril 1987)

\begin{abstract}
Résumé. - Des céramiques composites à base de superconducteur ionique NASICON $\left(\mathrm{Na}_{3} \mathrm{Zr}_{2} \mathrm{Si}_{2} \mathrm{PO}_{12}\right)$ et de verre peu conducteur $\left(\mathrm{Na}_{2} \mathrm{Zr}_{4} \mathrm{Si}_{16} \mathrm{P}_{8} \mathrm{O}_{61}\right)$ ont été élaborées suivant une filière «SOL-GEL » par frittage à basse température. Les microstructures obtenues ont été analysées par microscopie électronique et l'inter-réaction entre les deux phases a été étudiée par microanalyse Raman. La conductivité des composites a été analysée par spectrométrie d'impédances complexes pour diverses proportions de verre (entre 3 et $90 \%$ pondéral) et pour différents degrés de réactions (découlant des différentes températures de frittage). Le seuil de percolation se situe vers $5 \%$ et, dès $10 \%$ de verre, les propriétés du composite sont déterminées par le verre. Une anomalie de conductivité est observée pour les composites contenant $3 \%$ de verre.
\end{abstract}

\begin{abstract}
NASICON $\left(\mathrm{Na}_{3} \mathrm{Zr}_{2} \mathrm{Si}_{2} \mathrm{PO}_{12}\right) /$ low conductor $\left(\mathrm{Na}_{2} \mathrm{Zr}_{4} \mathrm{Si}_{16} \mathrm{P}_{8} \mathrm{O}_{61}\right.$ glass) composites ceramics have been synthesized using sol-gel process and low-temperatures sintering. The microstructures have been studied by scanning electron microscopy and the chemical reaction between NASICON grains and the intergranular glassy second phase has been analysed using Raman Microprobe spectroscopy. Conductivity has been measured by impedance spectroscopy for various glass content between 3 and $90 \%$ (by weight) and for various degrees of reactions between NASICON and glass. The percolation threshold is about $5 \%$ and up $10 \%$ the properties are similar to those of the low conducting phase. An conductivity anomaly is observed for $3 \%$ glass content.
\end{abstract}

\section{Introduction.}

Les composés de type NASICON (un acronyme pour $\mathrm{Na}$ Super Ionic Conductor correspondant à la solution solide $\left.\mathrm{NaZr}_{2}\left(\mathrm{PO}_{4}\right)_{3}-\mathrm{Na}_{4} \mathrm{Zr}_{2}\left(\mathrm{SiO}_{4}\right)_{3}\right)$ sont très étudiés du fait de leurs remarquables propriétés électriques : $\sigma_{300}{ }^{\circ} \mathrm{C} \neq 10^{-1} \Omega^{-1} \mathrm{~cm}^{-1}$ pour la composition $\mathrm{Na}_{3} \mathrm{Zr}_{2} \mathrm{Si}_{2} \mathrm{PO}_{12}$ [1]. Sous forme de céramiques, le NASICON peut être utilisé dans différents dispositifs électrochimiques: accumulateurs, capteurs... $[2,3]$. La synthèse de céramiques parfaitement pures sur le plan cristallographique est difficile et certains problèmes rencontrés dans l'utilisation de cet électrolyte solide peuvent être reliés à la présence de phases parasites aux joints de grains ; celles-ci sont généralement soit de la zircone monoclinique, soit une phase vitreuse, riche en silice $[4,5]$; cette dernière phase est particulièrement importante dans les compositions déficitaires en zirconium [6]. Ces phases parasites proviennent soit de la réaction incomplète des matières premières, soit d'une carac- téristique du diagramme de phase : environ $100{ }^{\circ} \mathrm{C}$ avant la fusion incongruente de la phase

$$
\text { NASICON }\left(\mathrm{Na}_{2+x+4 y} \mathrm{Zr}_{2-y} \mathrm{Si}_{x} \mathrm{P}_{3-x} \mathrm{O}_{12}\right)
$$

une démixtion partielle peut se produire pour les compositions $x \neq 2, y>0$ [7]. Ces problèmes peuvent être évités en utilisant une filière de synthèse «SOL-GEL » $[8,9]$.

Pour une meilleure compréhension des propriétés électriques de l'électrolyte solide $\mathrm{Na}_{3} \mathrm{Zr}_{2} \mathrm{Si}_{2} \mathrm{PO}_{12}$, nous avons volontairement préparé des céramiques composites contenant des proportions variables de verre (la composition choisie, $\mathrm{Na}_{2} \mathrm{Zr}_{4} \mathrm{Si}_{16} \mathrm{P}_{8} \mathrm{O}_{61}$, est proche de celle qui se forme spontanément pour les compositions déficitaires en zirconium [9]). La méthode de synthèse utilisée permet d'enrober les grains de NASICON par la phase vitreuse. En effet, s'il est bien établi qu'à une interface conducteur ionique/conducteur électronique des phénomènes de double-couche se produisent et permettent de réali- 
ser des super-condensateurs [10], il a été avancé que des phénomènes physiques à l'interface superconducteur ionique/isolant pouvaient conduire à une élévation de la conductivité [11]. Cependant, les exemples étudiés (comme par exemple les mélanges LiI $/ \mathrm{Al}_{2} \mathrm{O}_{3} \gamma$ ) sont peu convaincants du fait de la possibilité d'obtenir à l'interface une seconde phase conductrice (par exemple un hydrate, voire une solution ionique) du fait de la présence d'espèces protoniques. Aussi il nous a semblé intéressant d'étudier les propriétés électriques de composites susperconducteurs-phase isolante à base de NASICON.

\section{Expérimental.}

2.1 SYNThÈSE. - La figure 1 résume la filière de synthèse. La phase pure $\mathrm{Na}_{3} \mathrm{Zr}_{2} \mathrm{Si}_{2} \mathrm{PO}_{12}$ est d'abord préparée par une méthode sol-gel intitulée «méthode 1 », largement décrite dans la référence [9]: une solution de propoxide de zirconium (AlfaVentron) dans le propanol (environ 1/1 en volume) est hydrolysée simultanément par deux solutions aqueuses, respectivement une solution colloïdale de

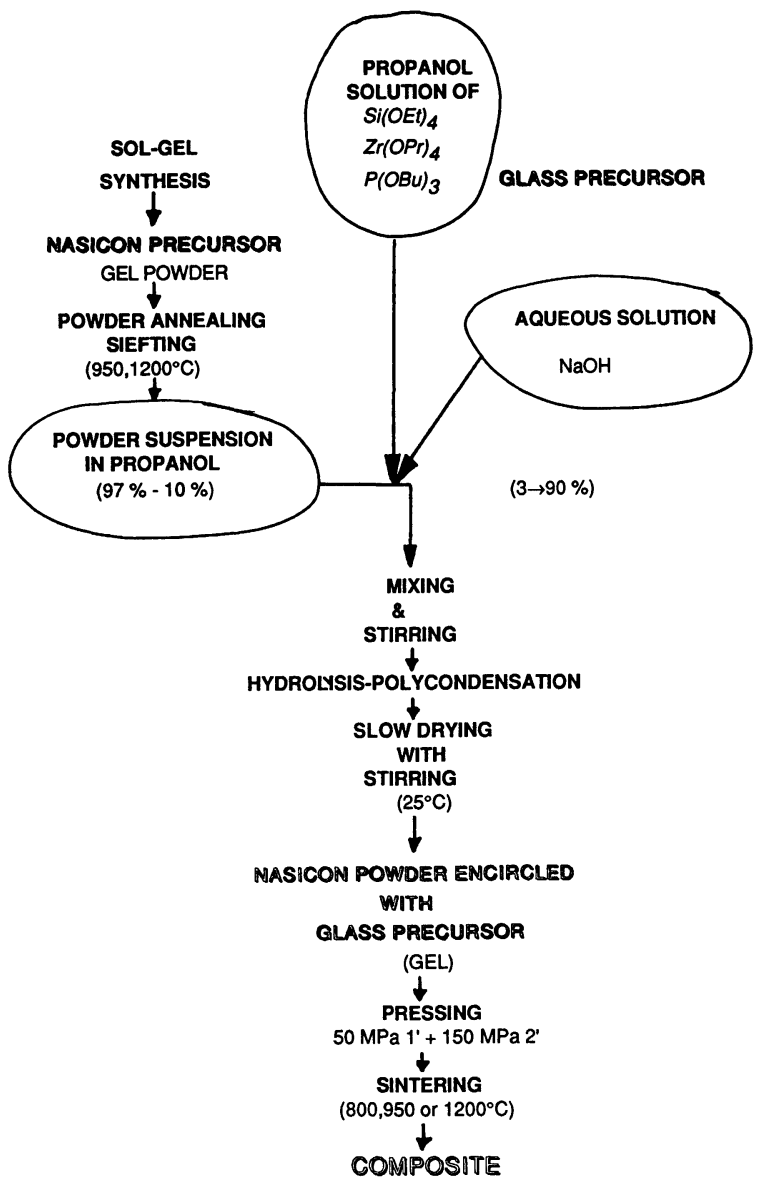

Fig. 1. - Schéma général de synthèse de céramiques composites.

[General flowchart of composite ceramics process.] silicate de soude $\left(\mathrm{Na}_{2} \mathrm{O} 3 \mathrm{SiO}_{2} 3 \mathrm{H}_{2} \mathrm{O}\right.$, Prolabo) et une solution de $\mathrm{NH}_{4} \mathrm{H}_{2} \mathrm{PO}_{4}$ (Prolabo), une agitation vigoureuse étant réalisée. Les quantités d'eau utilisées correspondent à environ 10 moles d'eau par atome d'oxygène d'alkoxyde. Après séchage à température ambiante une poudre fine, formée de particules d'environ $0,1 \mu \mathrm{m}$ est obtenue. Afin d'éviter la formation d'agglomérats durs, le gel est désagrégé continuellement pendant son séchage. Un tamisage dynamique [13] est effectué pour parfaire l'homogénéité dimensionnelle de la poudre. Le gel obtenu peut être représenté par la formule $\mathrm{Na}_{3} \mathrm{Zr}_{2} \mathrm{Si}_{2} \mathrm{PO}_{12}, 6,3 \mathrm{H}_{2} \mathrm{O}$; il est amorphe [13]. La poudre est ensuite traitée thermiquement à 800,950 ou $1200^{\circ} \mathrm{C}$ afin de développer trois états différents de la structure NASICON [7]. Après ces recuits la taille des particules est comprise entre 0,1 et $0,5 \mu \mathrm{m}$.

La structure NASICON peut rapidement être décrite comme formée de tétraèdres isolés $\left(\mathrm{SiO}_{4}, \mathrm{PO}_{4}\right)$ définissant entre eux des cavités soit vides, soit occupées par les ions tétra- ou monovalents. Les tétraèdres présentent un fort désordre d'orientation statique et/ou dynamique, désordre couplé à des degrés de remplissage variables des sites, ce désordre jouant un rôle important pour la diffusion rapide des ions alcalins [7]. Si le traitement thermique reste inférieur à $900^{\circ} \mathrm{C}$, le NASICON préparé par méthode sol-gel est amorphe, certains tétraèdres étant associés et les chemins de conduction mal définis. Vers $950{ }^{\circ} \mathrm{C}$, la structure NASICON se forme, mais un fort désordre d'orientation statique reste présent. Recuit au-dessus de $1150^{\circ} \mathrm{C}$, le matériau présente des transitions bien définies, en particulier la transition monoclinique/rhombohédrique à $150^{\circ} \mathrm{C}$ déclenche un fort désordre dynamique d'orientation des tétraèdres et l'état superconducteur ionique apparaît : l'énergie d'activation de la conductivité est alors inférieure à $0,15 \mathrm{eV}$ [7].

Le verre pur est préparé suivant la «méthode 2 » [9] : une solution alcoolique des alkoxides de $\mathrm{Si}, \mathrm{Zr}$ et $\mathrm{P}\left(\mathrm{Si}\left(\mathrm{OC}_{2} \mathrm{H}_{5}\right)_{4}, \mathrm{Zr}\left(\mathrm{O}_{4} \mathrm{C}_{3} \mathrm{H}_{7}\right)_{4}, \mathrm{P}\left(\mathrm{O}_{t} \mathrm{C}_{4} \mathrm{H}_{5}\right)_{3}\right.$, AlfaVentron) est hydrolysée par une solution aqueuse de $\mathrm{NaOH}$, une forte agitation étant là aussi nécessaire.

Pour obtenir la poudre qui donnera par traitement thermique la céramique composite NASICONVERRE, la poudre de NASICON, préalablement traitée thermiquement à 800,950 ou $1200^{\circ} \mathrm{C}$, conservée sèche, est mise en suspension dans le propanol puis mélangée avec la solution de propanol contenant les alkoxides de $\mathrm{Zr}$, Si et P (Fig. 2). La solution obtenue, agitée constamment pour conserver la poudre en suspension, est alors hydrolysée par $\mathrm{NaOH}$. Un fort excès d'eau (50 à 100 moles d'eau pour 1 mole de verre et une quantité minimale d'alcool (1 volume de propanol pour 1 volume d'alkoxide) sont utilisés. En effet, l'excès d'eau permet d'obtenir un gel très proche d'un oxide [13], ce qui limite le retrait provoqué par le départ des 


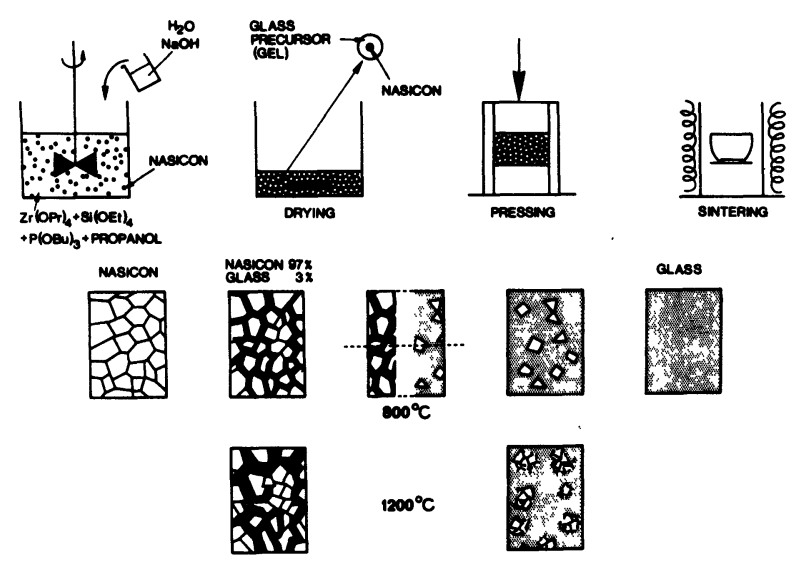

Fig. 2. - Représentation schématique des microstructures désirées et de la méthode d'enrobage des grains de NASICON par le précurseur de verre. En fonction de la température à laquelle est effectué le frittage, la phase intergranulaire peut être du verre pur ou bien un produit de réaction entre celui-ci et le NASICON.

[Schematic view of the expected microstructures and of the method for the glass/NASICON grains encircling. As a function of the sintering temperature, the intergranular phase can be pure glass or a new phase made from NASICON and glass reaction.]

espèces volatiles entre 100 et $400{ }^{\circ} \mathrm{C}$. Après séchage à $50^{\circ} \mathrm{C}$ (là aussi le gel doit être constamment désagrégé pendant le séchage) la poudre est compactée suivant un cycle court : $50 \mathrm{MPa}, 1 \mathrm{~min}+$ $150 \mathrm{MPa}, 2 \mathrm{~min}$, décompression $2 \mathrm{~min}$. Le gel précurseur de verre étant hygroscopique, le pressage doit être fait aussitôt après séchage. La pastille obtenue est frittée à 800,950 ou $1200^{\circ} \mathrm{C}$ pendant $1 \mathrm{~h}$ (vitesse de chauffe $100^{\circ} \mathrm{C} / \mathrm{h}$, refroidissement $300^{\circ} \mathrm{C} / \mathrm{h}$ ).

\subsection{Moyens D'INVESTIGATION. - La microanalyse}

Raman est effectuée avec un instrument MOLE I.S.A. associé à un laser Argon Spectra Physics. La longueur d'onde excitatrice est $514,5 \mathrm{~nm}$ avec une puissance de $50 \mathrm{~mW}$. Le volume analysé est d'environ 1 à $5 \mu \mathrm{m}^{3}$. L'analyse dilatométrique est obtenue avec un appareil ADAMEL. La microscopie électronique est faite sur un appareil HITACHI. Les mesures électriques sont faites à l'aide d'un analyseur de réponse en fréquence HP $4192 \mathrm{~A}$ piloté par un micro-ordinateur Olivetti M24. Les électrodes (déposées sur les échantillons frittés) sont constituées d'une laque d'argent séchée à $150^{\circ} \mathrm{C}$ ou d'une laque platine frittée à $800^{\circ} \mathrm{C}$. La diffusion de l'argent dans la céramique peut être considérée comme nulle dans la gamme de températures explorée par les mesures $\left(20-600{ }^{\circ} \mathrm{C}\right)$.

\section{Microstructures.}

3.1 Topologie. - La figure 3 compare les courbes de dilatation de la composition $\mathrm{Na}_{3} \mathrm{Zr}_{2} \mathrm{Si}_{2} \mathrm{PO}_{12}$, du REVUE DE PHYSIQUE APPLIQUÉE. - T. 22, N` 8, AOUT 1987

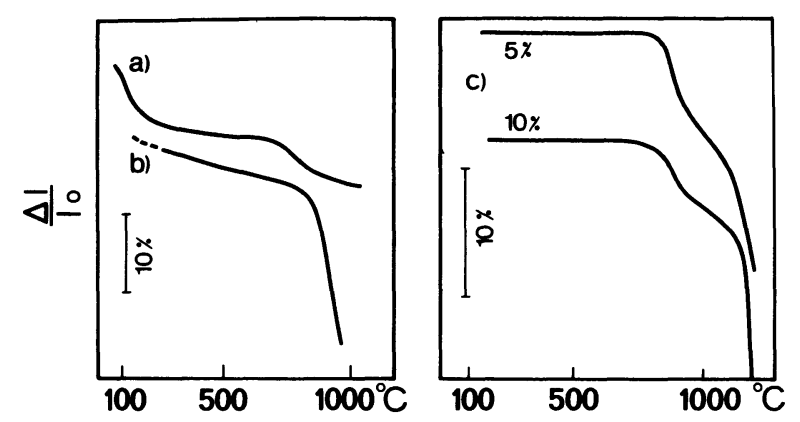

Fig. 3. - Courbes dilatométriques $\left(\Delta l / l_{0}\right)$ : a) NASICON, b) verre, c) composites avec respectivement 5 et $10 \%$ de verre (les grains de NASICON ont été préalablement traités à $950^{\circ} \mathrm{C}$ ).

[Dilatometric traces $\left(\Delta l / l_{0}\right)$ : a) NASICON, b) glass, c) 5 and $10 \%$ glass composites (NASICON grains have been previously heated at $950^{\circ} \mathrm{C}$ ).]

verre $\mathrm{Na}_{2} \mathrm{Zr}_{4} \mathrm{Si}_{16} \mathrm{P}_{8} \mathrm{O}_{61}$ et de deux mélanges contenant $5 \%$ et $10 \%$ de verre respectivement. Tous présentent un retrait en dessous de $400{ }^{\circ} \mathrm{C}$ du fait du départ des solvants et des espèces protonées lors de la transformation gel $\rightarrow$ oxide amorphe $[7,9,13]$. La densification du NASICON se produit entre 700 et $900{ }^{\circ} \mathrm{C}$ tandis que le verre montre un retrait sans fin au-dessus de $850^{\circ} \mathrm{C}$ du fait de l'abaissement de sa viscosité. L'ajout de $5 \%$ de verre conduit déjà à l'apparition de ce comportement, mais un palier de réaction est observé entre 900 et $1100^{\circ} \mathrm{C}$. Les phénomènes sont d'autant plus nets que l'ajout est plus important. L'action d'une seconde phase vitreuse est donc très favorable au frittage. Aussi dans les premières études $[5,14,15]$, peu d'attention fut portée à cette phase vitreuse car elle améliorait le frittage et ainsi dans une certaine mesure les propriétés électriques en courant alternatif. Par contre l'effet est dramatique pour les applications nécessitant un transport des ions dans la céramique (accumulateur) mais aussi pour la stabilité vis-à-vis du sodium fondu [16]. Certains auteurs $[17,18]$ relièrent cependant l'existence de différentes relaxations dans les spectres d'impédance complexe à la présence de secondes phases (zircone, phases indéterminées...).

La figure 4 compare les microstructures observées sur fractures pour une même concentration de verre (5\%) en fonction de la température de recuit et pour une même température $\left(950^{\circ} \mathrm{C}\right)$ en fonction du pourcentage de verre. En accord avec les analyses dilatométriques, on note qu'en dessous de $950{ }^{\circ} \mathrm{C}$, la réaction entre le verre et les grains de NASICON reste faible. La durée du cycle de compaction (quelques min) a été volontairement limitée pour prévenir une compaction complète (un cycle de 15 min est nécessaire pour une compaction optimale $[13,20])$ rendant plus difficile l'observation des modifications. On peut supposer que cette compaction incomplète augmentera la quantité de verre 


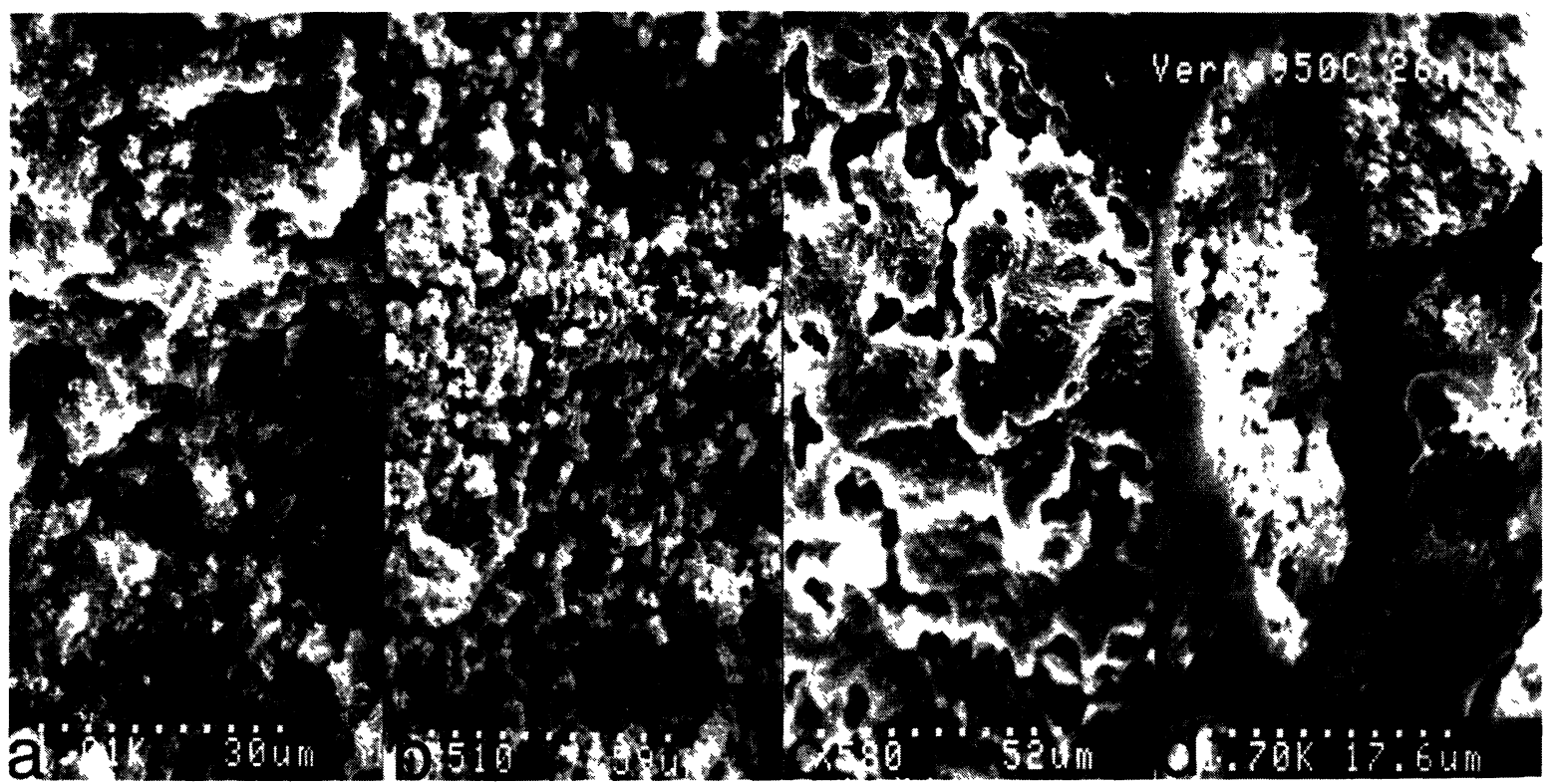

Fig. 4. - Microphotographies de fractures de céramiques composites à $5 \%$ de verre, frittés respectivement à 800 (a), 950 (b) et $1150^{\circ} \mathrm{C}$ (c). En comparaison le cas d'un composite à $60 \%$ de verre, fritté à $950^{\circ} \mathrm{C}$ est montré (d). (Echelles et grossissements sont indiqués en bas de la photographie).

[Photomicrographs of $5 \%$ glass composites (fractures) sintered at 800 (a), 950 (b) and $1150{ }^{\circ} \mathrm{C}$ (c), respectively. For comparison, the photomicrograph of a $60 \%$ glass composite $\left(950^{\circ} \mathrm{C}\right.$ sintered) is shown (d). (Magnification and scale are noted below).]

nécessaire à la modification des propriétés électriques. A $800{ }^{\circ} \mathrm{C}$ la taille moyenne des grains est inférieure à $0,5 \mu \mathrm{m}$ et la cassure reste intergranulaire. Après frittage à $950^{\circ} \mathrm{C}$ un fort grossissement de la microstructure apparaît. Les grains ont entre 2 et $10 \mu \mathrm{m}$ comme dans les céramiques monophasées [20] ; la taille des pores grossit également. Tout ceci indique une réaction liée à la présence d'une phase liquide. Après frittage à $1150^{\circ} \mathrm{C}$, la microstructure est typique d'un frittage en phase liquide avec des pores de forme ronde et une cassure transgranulaire. La réaction reste cependant faible au-dessous de $950^{\circ} \mathrm{C}$ et se limite à la zone de contact VERRE/NASICON, car même pour une concentration de $60 \%$ de verre, la céramique reste bien hétérogène. La topologie observée correspond à ce qui est symbolisé dans la figure 2 .

\subsection{RÉACTIONS CHIMIQUES ET MICROHÉTÉROGÉ-} NÉITÉ. - La figure 5 compare les spectres Raman de composites frittés à 950 et $1150{ }^{\circ} \mathrm{C}$ pour différents taux de verre entre 5 et $95 \%$ (la forte diffusion observée en dessous de $200 \mathrm{~cm}^{-1}$ est principalement due à l'appareillage dont le taux de réjection de la lumière incidente est faible). Le spectre du composite à $5 \%$ de verre est très comparable à celui du NASICON pur [7], si ce n'est la large bosse entre 600 et $1300 \mathrm{~cm}^{-1}$ due à la présence du verre. Un ajout plus important modifie le spectre du massif $\nu \mathrm{P}-\mathrm{O} / \nu \mathrm{Si}-\mathrm{O}$ (modes d'élongation des tétraèdres) avec une augmentation de l'intensité du mode de vibration $\nu \mathrm{P}-\mathrm{O}$ à $1030 \mathrm{~cm}^{-1}$, ce qui traduit un déplacement de la composition vers $x=0$ [19]. Simultanément la bosse due au verre devient plus intense, avec en particulier une bande vers $800 \mathrm{~cm}^{-1}$ caractéristique de tétraèdres associés. ( $v \mathrm{P}-\mathrm{O}-\mathrm{P}$ ou $\nu \mathrm{Si}-\mathrm{O}-\mathrm{Si}$ ) [7]. Cependant jusqu'à la proportion de $60 \%$, on peut considérer que l'on a encore un composite contenant pour une proportion significative la phase NASICON ; cependant sa stœchiométrie évolue vers des compositions riches en phosphate $(x \rightarrow 1)$. Un traitement thermique à $1150^{\circ} \mathrm{C}$ conduit comme dans le cas des compositions voisines de $x=2$ à l'apparition de traces de zircone monoclinique (raies fines à 175-185, 335, 345, 380, 475, 573 et $\left.635 \mathrm{~cm}^{-1}\right)$. Les autres modifications résultant de l'ajout de verre ne sont pas fortement modifiées.

La figure 6 compare les spectres Raman obtenus pour deux localisations distantes d'environ 5-10 $\mu \mathrm{m}$ pour une céramique frittée à $950^{\circ} \mathrm{C}$ : la proportion de verre et la modification de la composition du NASICON sont plus marquées en a qu'en $b$, traduisant ainsi que la microhétérogénéité structurale est du même ordre que la taille des grains, en accord avec la représentation de la figure 2 .

\section{Propriétés électriques.}

4.1 CÉRAMiques « MONOPHASÉES ». - L'impédance $Z$ d'une céramique recouverte de deux électrodes métalliques peut être étudiée en fonction de la fréquence (entre $100 \mathrm{~Hz}$ et $10 \mathrm{MHz}$ dans ce travail). 

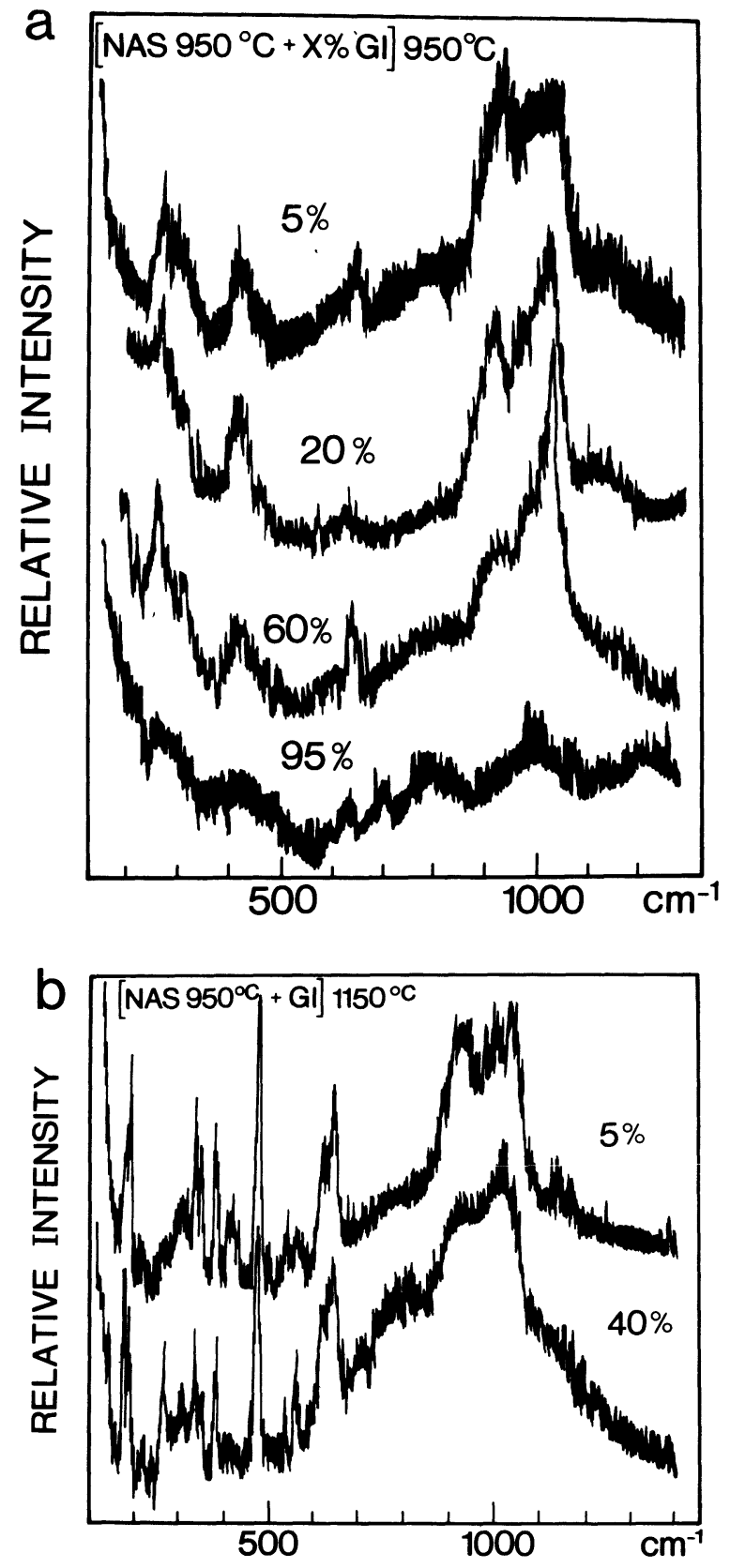

Fig. 5. - Spectres Raman enregistrés sur un volume localisé $\left(\sim 5 \mu \mathrm{m}^{3}\right)$ pour des composites frittés à 950 (a) ou $1150{ }^{\circ} \mathrm{C}(\mathrm{b})$. Les grains de NASICON ont été préalablement traités à $950^{\circ} \mathrm{C}$. Les proportions de verre varient entre 5 et $95 \%$.

[Microprobe Raman spectra of 950 (a) and $1150^{\circ} \mathrm{C}$ (b) sintered composites (the probe volume is $\sim 5 \mu \mathrm{m}^{3}$ ). The NASICON grains are previously thermally treated at $950{ }^{\circ} \mathrm{C}$. Various glass content between 5 and $95 \%$ are shown.]

Cette grandeur peut être représentée dans le plan complexe : $Z_{(\omega)}=R_{(\omega)}-i X_{(\omega)} ; R_{(\omega)}$ est la partie réelle, homologue à une résistance pure et $X_{(\omega)}$ la partie imaginaire, homologue à une capacité pure. Selon la nature des électrodes (interface conducteur ionique/conducteur électronique parfaitement blo-

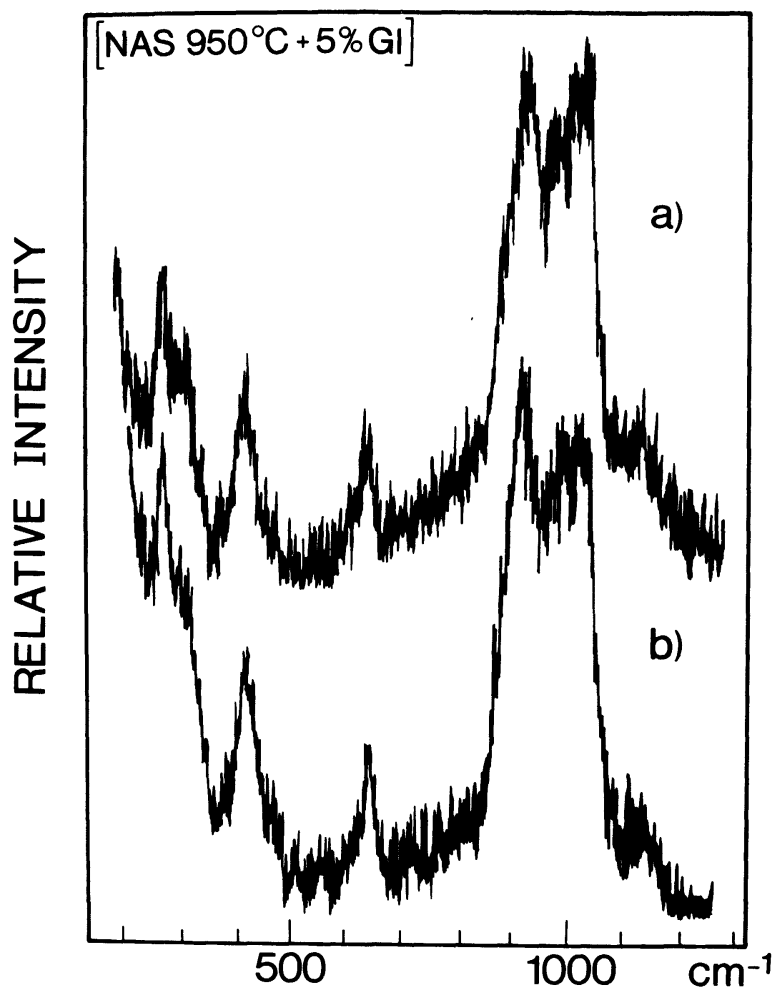

Fig. 6. - Spectres Raman obtenus pour une céramique contenant $5 \%$ de verre et frittée à $950^{\circ} \mathrm{C}$. Le spectre est obtenu pour deux localisations ( $a$ et $b$ ) distantes d'environ $10 \mu \mathrm{m}$.

[Microprobe Raman spectra of a $5 \%$ glass composite ( $950{ }^{\circ} \mathrm{C}$ sintered). The distance between the two analysed places is about $10 \mu \mathrm{m}$.

quante ou non), la nature de la géométrie de l'interface (porosité, percolation) la nature de la céramique (monophasée ou non) et la nature des espèces mobiles de nombreux cas «typiques » de diagrammes $Z_{(\omega)}$ ont été définis et étudiés [21-27].

Dans le cas simple d'un «bon superconduteur ionique » associé à des électrodes bloquantes, le schéma équivalent est peu différent d'une capacité (la capacité de double couche) associée à la résistance de l'électrolyte (nous ne considérons pas ici les effets mineurs des câbles et connexions). En fait un écart à $\pi / 2$ est observé et l'on observe comme indiqué sur la figure $7 \mathrm{~b}$ pour une céramique de NASICON $x=2$, frittée à $1250^{\circ} \mathrm{C}$, et ayant des électrodes de $\mathrm{Pt}$, une droite faisant un angle de 80 à $86^{\mathrm{d}}$ selon les échantillons. L'intersection avec l'abscisse donne la résistance globale de l'échantillon et par là, la conductivité du matériau.

Dans le cas d'une électrode en Ag pour laquelle une adsorption spécifique, voire une insertion, peut survenir à l'interface (ce qui correspond à une électrode partiellement bloquante) le processus de diffusion mis en jeu induit une dépendance temporelle en $\sqrt{t}$ et dans l'espace des impédances une droite de pente $1 / 2$ (droite de Warburg). C'est ce qui 
a)

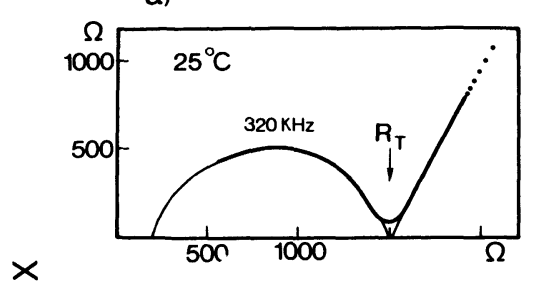

b)
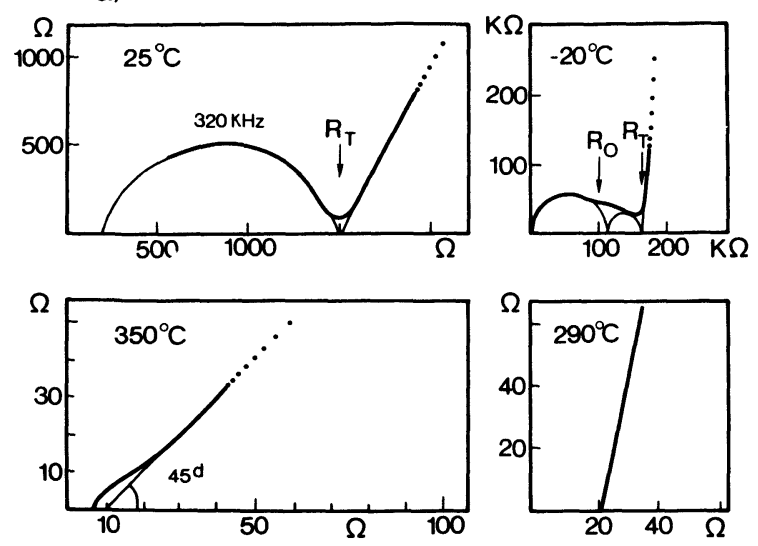

$\mathbf{R}$

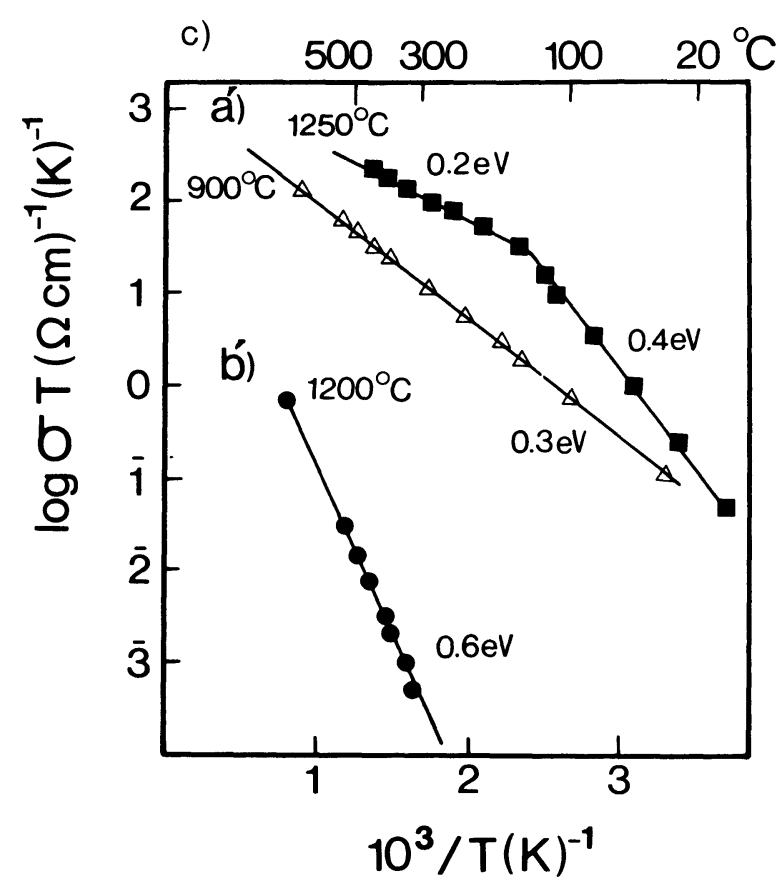

Fig. 7. - Diagrammes d'impédances de céramiques NASICON (monophasées) réalisées avec des électrodes d'argent (a) ou de platine (b). Les céramiques ont été frittées respectivement à 950 (a) et $1250^{\circ} \mathrm{C}$ (b). Les conductivités du NASICON (fritté à 900 et $1250^{\circ} \mathrm{C}$, $\mathrm{a}^{\prime}$ ) et du verre (fritté à $1200^{\circ} \mathrm{C}, \mathrm{b}^{\prime}$ ) sont donnés sur la figure (c) (représentation $\log _{10} \sigma T=f(1 / T)$ ).

[Impedances diagrams of «pure " NASICON ceramics with $\mathrm{Ag}$ (a) or $\mathrm{Pt}$ (b) electrodes for various temperatures. Samples are sintered at $950{ }^{\circ} \mathrm{C}$ (a) ou $1250{ }^{\circ} \mathrm{C}$ (b), respectively. Corresponding conductivity plots $\left(\log _{10} \sigma T=f(1 / T)\right)$ are given on figure (c) for NASICON $\left(a^{\prime}\right)$ and glass $\left(b^{\prime}\right)$. The sintering temperature are given.]

est effectivement observé pour un spécimen $\mathrm{Ag} / \mathrm{NASICON} / \mathrm{Ag}$.

Lorsque l'on abaisse la température de l'échantillon, d'autres phénomènes de relaxation deviennent visibles. Ces relaxations (par exemple celle observée à $320 \mathrm{kHz}$ sur la Fig. 7a) sont généralement attribuées soit à la présence de secondes phases, soit même aux joints de grains de la céramique. Des effets de charge d'espace non directement attribuables peuvent aussi être invoqués. Les meilleurs critères d'attribution sont, à notre avis, l'énergie d'activation de ces phénomènes et leur corrélation avec les microstructures observées. Ainsi dans le cas de la figure 7, une des relaxations de l'échantillon fritté à $1250^{\circ} \mathrm{C}$ peut être attribuée à la zircone précipitée aux joints de grains en accord avec les analyses de diffraction RX et de diffusion Raman, et avec la valeur de l'énergie d'activation (voisine de $1 \mathrm{eV}$ ). Par contre dans le cas de l'échantillon fritté à $950^{\circ} \mathrm{C}$ qui apparaît homogène pour les différentes techniques d'analyses utilisées une attribution précise est plus difficile : la seule "inhomogénéité » reconnue est la présence de cristallites de NASICON dans la matrice amorphe de même composition [28]. La figure 7c compare les évolutions de la conductivité (selon la représentation $\log \sigma T=f(1 / T)$ ) pour des céramiques de NASICON $\left(\mathrm{Na}_{3} \mathrm{Zr}_{2} \mathrm{Si}_{2} \mathrm{PO}_{12}\right)$ « pures », frittées à 900 et $1250^{\circ} \mathrm{C}$ ainsi que pour le verre cuit à $1200^{\circ} \mathrm{C}$. Les valeurs mesurées sur ces échantillons seront considérées dans la suite du travail comme les valeurs de référence des composés purs, en accord avec les résultats antérieurs [4-9].

4.2 CÉramiques COMPosites. - La figure 8 compare les diagrammes d'impédances de composites frittés à 800 et $950^{\circ} \mathrm{C}$ avec respectivement 3,5 et $10 \%$ de verre, à ceux des deux composés limites (à basse et haute fréquence les points expérimentaux sont suffisamment rapprochés pour former une courbe continue épaisse ; les courbes fines correspondent aux déconvolutions). De même que pour le composé NASICON supposé monophasé, le diagramme se compose d'une droite à $45^{\mathrm{d}}$ type Warburg se poursuivant vers les hautes fréquences par des arcs de cercles, plus ou moins bien centrés sur l'axe. Pour une même température, au fur et à mesure que la proportion de verre augmente, la part des arcs de cercle augmente, ainsi que le temps caractéristique associé à ces relaxations. Pour les faibles taux de verre la gamme de fréquence pour laquelle la droite de Warburg est observée est large $(230 \mathrm{~Hz}-1 \mathrm{MHz})$. Elle se réduit fortement, pour les teneurs moyennes, mais on peut supposer que la faible portion de droite observée est de même nature. Le verre pur montre un diagramme identique à ce qui est observé dans le cas d'un double phénomène d'absorption et de diffusion, avec une pente à $45^{\mathrm{d}}$ près du pt 0,0 [24-27]. Dans ces diagrammes de composites il semble donc cohérent d'attribuer les relaxations observées du côté basses fréquences à la seconde phase vitreuse.

Le schéma équivalent le plus simple pour rendre compte des diagrammes est une résistance $R_{\mathrm{M}}$ en parallèle avec la capacité intergrain, le tout en série 

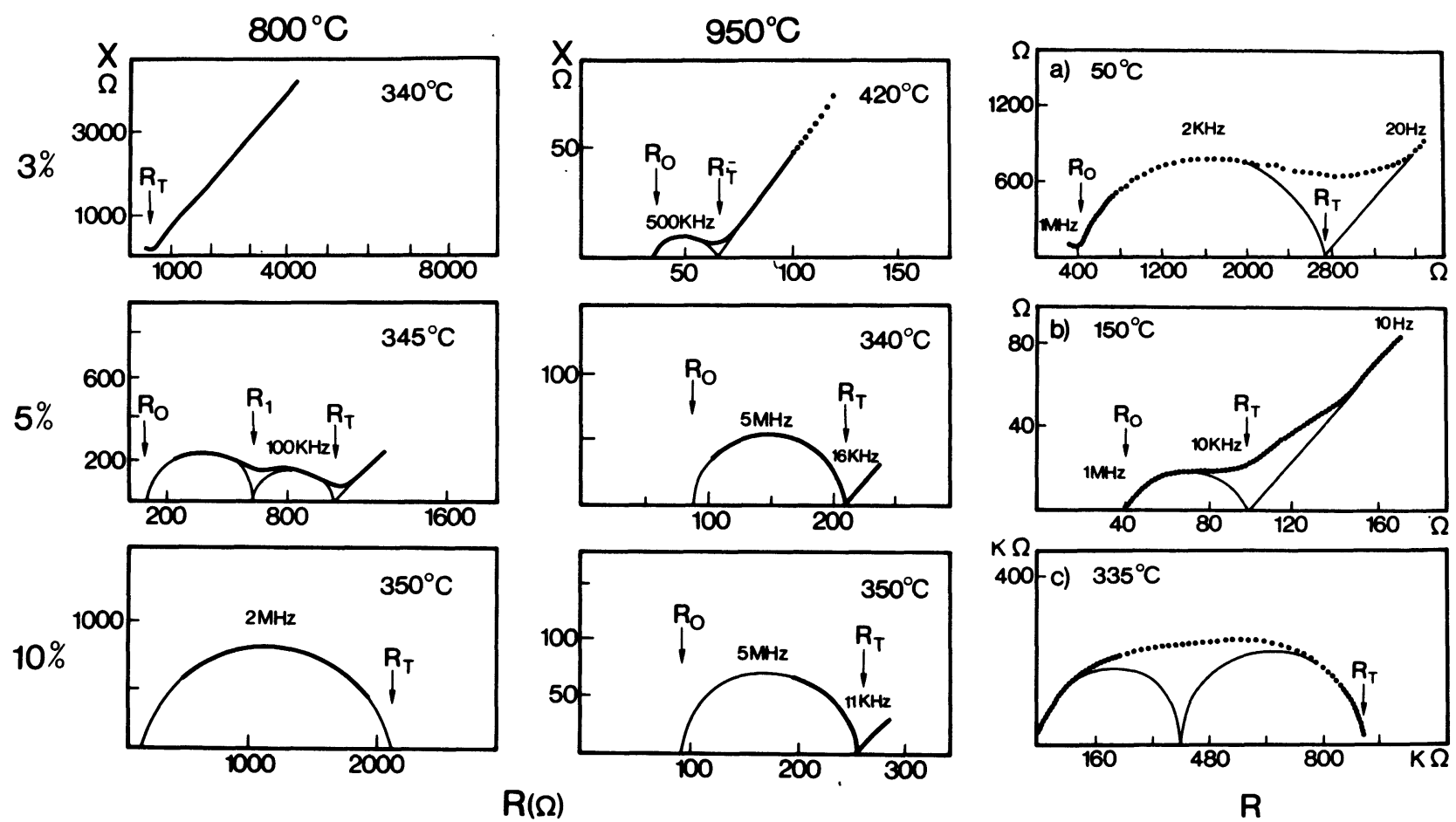

Fig. 8. - Diagrammes d'impédances de composites à 3,5 et $10 \%$ de verre, frittés à 800 ou $950^{\circ} \mathrm{C}$ à différentes températures (électrodes d'argent). Les grains de NASICON ont été traités thermiquement à $950^{\circ} \mathrm{C}$. Les diagrammes obtenus pour les céramiques de NASICON (fritté à $950^{\circ} \mathrm{C}$ ) a, b) et de verre (fritté à $1200^{\circ} \mathrm{C}$ ), c) sont donnés en comparaison.

[Impedance diagrams of 3,5 and $10 \%$ glass composites (sintered at 800 or $950{ }^{\circ} \mathrm{C}, \mathrm{Ag}$ electrodes). The NASICON grains have been previously treated at $950^{\circ} \mathrm{C}$. For comparison, the diagrams obtained with pure NASICON $\left(950^{\circ} \mathrm{C}\right.$ sintered, $\mathrm{a}, \mathrm{b})$ and pure glass $\left(1200^{\circ} \mathrm{C}\right.$ sintered, c) are given.]

avec la résistance $R_{0}$ [24-25]; dans ce schéma équivalent la résistance totale est $R_{\mathrm{T}}=R_{0}+R_{\mathrm{M}}$, $R_{0}$ étant attribué à la résistance de la seconde phase et $R_{\mathrm{M}}$ la résistance des grains de matériaux. Dans le cas du phénomène de Warburg l'intersection avec l'axe des abscisses correspond à la résistance $R_{\mathrm{T}}$ [27]. Les cas intermédiaires peuvent être décrits par l'association des deux cas limites. A partir de ces modèles simples, il est possible de déterminer l'évolution de la conductivité totale du matériau déduite de la résistance $R_{\mathrm{T}}$; lorsque deux phénomènes de relaxation sont visibles la conductivité totale $R_{\mathrm{T}}$, la conductivité intrinsèque du matériau $R_{\mathrm{M}}=R_{\mathrm{T}}-R_{0}$ et celle de la seconde phase $R_{0}$ peuvent être simultanément déterminées). La représentation en loi d'Arrhénius $\sigma T=f(1 / T)$ permet de visualiser directement l'énergie d'activation $E_{\mathrm{A}}\left(\sigma T=\sigma_{0} \exp -E_{\mathrm{A}} / k T\right)$. On suppose alors que la relation entre $\sigma$ et $R$ peut se faire suivant $R=e / \sigma S$ où $e$ est l'épaisseur de matériau entre électrodes (comprise entre 0,5 et $3 \mathrm{~mm}$ pour nos échantillons) et $S$ la surface (comprise entre 15 et $50 \mathrm{~mm}^{2}$ ). Ceci est parfaitement exact pour un échantillon homogène, aux surfaces lisses, mais sans doute erroné pour un composite dont la géométrie de percolation est complexe. La figure 9 compare les conductivités déduites de $\boldsymbol{R}_{\mathrm{T}}$ pour différents composites, frittés à 800 ou $950^{\circ} \mathrm{C}$. On observe bien que, grossièrement, les courbes des composites sont comprises entre celles des deux composés limites. On note cependant que :

1) dès que l'ajout de verre atteint $10 \%$, les propriétés électriques « macroscopiques » sont plus

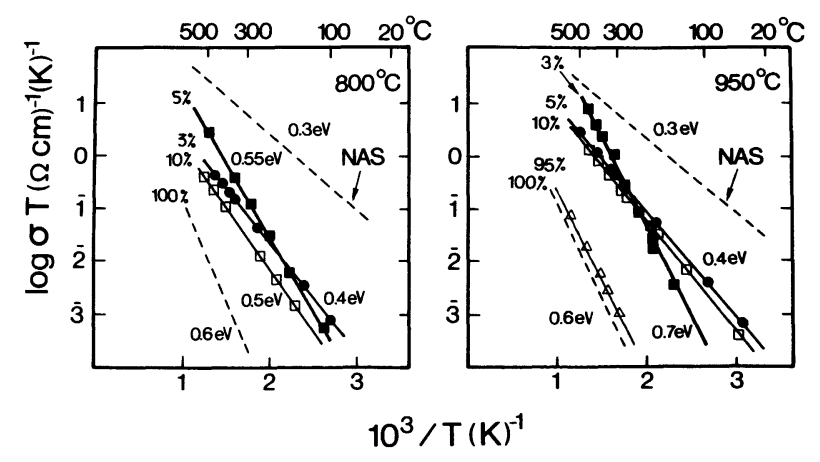

Fig. 9. - Conductivité totale (déduite de $R_{\mathrm{T}}$ ) pour des échantillons frittés à 800 et $950^{\circ} \mathrm{C}$ et contenant différents pourcentages de verre (0 à $100 \%)$.

[Total conductivities (deduced from $R_{\mathrm{T}}$ ) of 800 or $950{ }^{\circ} \mathrm{C}$ sintered ceramics containing various glass content ( 0 to $100 \%)$.] 

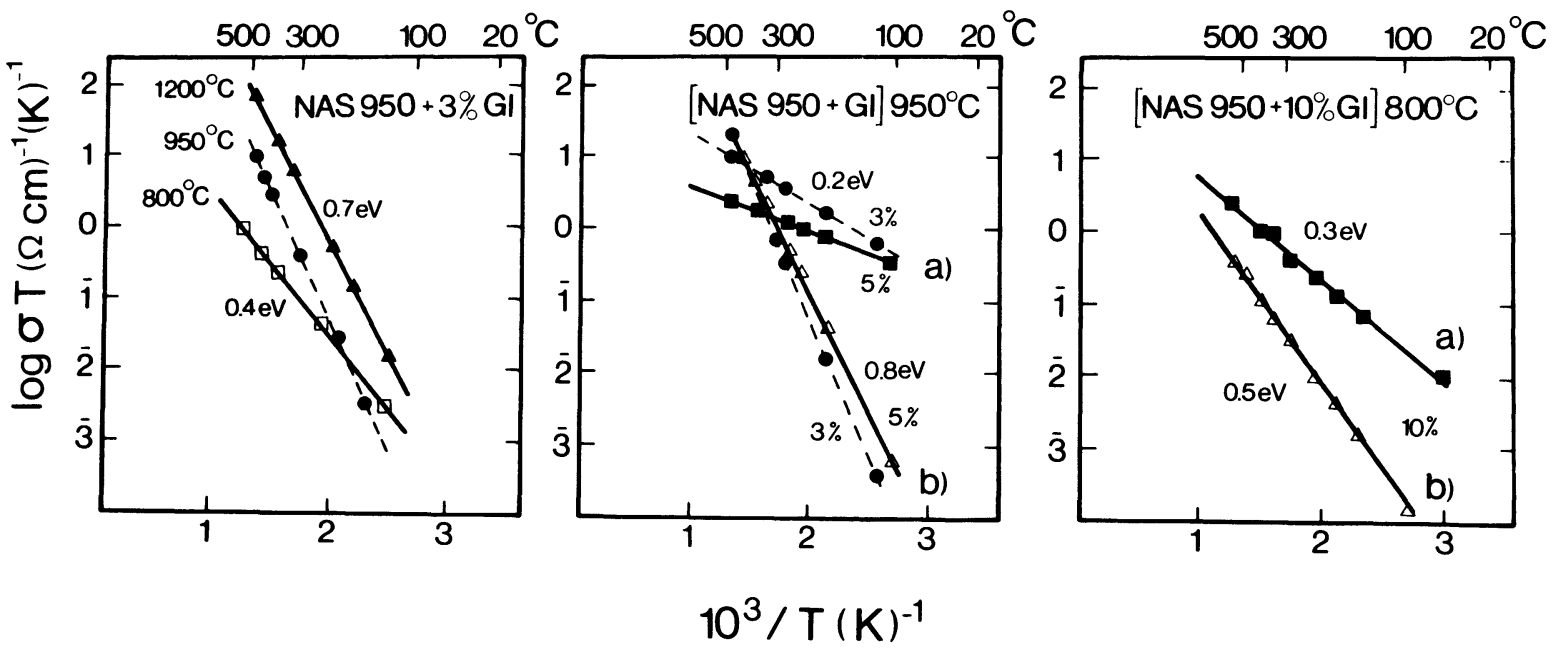

Fig. 10. - Evolution de la conductivité pour un composite à $3 \%$ de verre en fonction de la température $(800,950$, $1200^{\circ} \mathrm{C}$ ) et comparaison des contributions granulaires (a) et intergranulaires (b) pour des composites à 3,5 et $10 \%$ de verre. Les grains de NASICON ont été préalablement traités à $950^{\circ} \mathrm{C}$.

[Conductivity plots of $3 \%$ glass composites sintered at 800,950 and $1250{ }^{\circ} \mathrm{C}$, and comparison between the bulk conductivity (a) and the second phase contribution (b) for 3, 5 and $10 \%$ glass composites.]

proches de celles du verre "mauvais conducteur" que de celles de la phase majoritaire (NASICON),

2) l'énergie d'activation évolue régulièrement de $0,3 \mathrm{eV}$ à $0,6 \mathrm{eV}$, sauf pour les composites à $3 \%$ (frittage $950^{\circ} \mathrm{C}$ ) et à $5 \%$ (frittage $800^{\circ} \mathrm{C}$ ),

3) l'énergie d'activation augmente légèrement avec la température de frittage, en accord avec la formation de compositions intermédiaires du fait de la réaction chimique entre le NASICON et le verre comme cela a été mis en évidence par diffusion Raman.

Si l'on compare la conductivité déduite de $R_{\mathrm{M}}=$ $R_{\mathrm{T}}-R_{0}$ à celle déduite de $R_{0}$, c'est-à-dire les relaxations attribuées respectivement au grain («matériau pur ») et à la seconde phase, on obtient deux comportements distincts: la première a une «faible » énergie d'activation $(\sim 0,2 \mathrm{eV})$, très proche de celle du NASICON "pur », et la seconde une énergie beaucoup plus forte $(\sim 0,8 \mathrm{eV})$, comparable à celle du verre pur. Au fur et à mesure que la proportion de verre, ou bien que la température de frittage s'élève, les deux énergies, mais aussi les conductivités, se rapprochent (Fig. 10). Ceci est en parfait accord avec l'existence d'une réaction entre les deux phases comme observée par diffusion Raman et microscopie. En fait, au-dessus de $10 \%$ de verre, la séparation entre les différentes relaxations devient difficile sur une gamme de température suffisamment large pour mesurer correctement l'énergie d'activation. Ceci peut être dû :

1) soit à une réaction trop forte pour conserver des comportements électriques distincts,

2) soit au fait que la résolution de notre appareillage est insuffisante. En effet, la meilleure résolution en spectroscopie d'impédance complexe est obtenue avec d'autres appareils (comme par exemple le Solartron Energetec) où il est possible de définir la référence qu'utilisera le pont pour faire sa mesure, afin qu'elle ait un comportement en fréquence le plus proche possible de l'échantillon $[29,30]$.

\section{Conclusion.}

L'élaboration de composites formés de deux matériaux ayant des propriétés électriques distinctes est

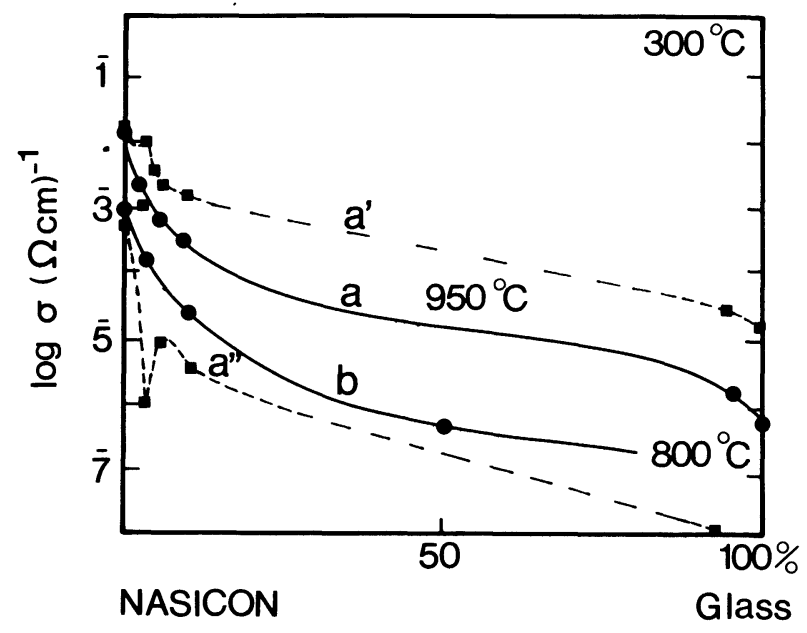

Fig. 11. - Evolution de la conductivité à $500{ }^{\circ} \mathrm{C}\left(\mathrm{a}^{\prime}\right)$, $300^{\circ} \mathrm{C}(\mathrm{a})$ et $100^{\circ} \mathrm{C}\left(\mathrm{a}^{\prime \prime}\right)$ de céramiques frittées à $950^{\circ} \mathrm{C}$ pour différents taux de verre. La conductivité à $300^{\circ} \mathrm{C}$ de céramiques frittées à $800^{\circ} \mathrm{C}$ est donnée en comparaison (b).

[Conductivity at $50{ }^{\circ} \mathrm{C}\left(\mathrm{a}^{\prime}\right), \quad 300{ }^{\circ} \mathrm{C}$ (a) and $100{ }^{\circ} \mathrm{C}$ $\left(a^{\prime \prime}\right)$ of $950^{\circ} \mathrm{C}$ sintered ceramics with vatious glass contents. The $300{ }^{\circ} \mathrm{C}$ conductivity of $800{ }^{\circ} \mathrm{C}$ sintered ceramics are given for comparison (b).] 
possible grâce à l'emploi des techniques SOL-GEL qui permettent un contrôle de l'hétérogénéité à l'échelle submicronique et un frittage à une température suffisamment basse pour limiter les réactions chimiques entre ces deux phases. Ceci ouvre la voie à la réalisation de microstructures complexes où topologie et propriétés électriques sont fortement liées (composés ferroélectrique, condensateurs type III, varistances, écran luminescents...) et par-là à une meilleure compréhension des propriétés liées aux interfaces. L'association de la microanalyse Raman et de la spectrométrie d'impédance conduit à une connaissance plus approfondie de la microstructure. Les propriétés électriques du grain superconducteur ionique et de la phase intergranulaire " isolante » peuvent être séparées pour des teneurs inférieures à $20 \%$, au-delà de laquelle la réaction entre les deux phases moyenne les propriétés électriques. Cependant, la prise en compte des résultats obtenus par l'ensemble des techniques d'analyses montre que pour $40 \%$ de verre et un recuit à $1150{ }^{\circ} \mathrm{C}$, les deux phases restent distinctes mais séparées par une phase de composition intermédiaire. Cette étude montre qu'il est nécessaire d'étudier les propriétés électriques de céramiques dans une large gamme de températures afin que la présence des secondes phases à l'état de traces soit décélée. Ainsi dans les céramiques NASICON que nous considérons comme pures d'après les autres méthodes d'analyse, les relaxations provoquées par des traces de zircone deviennent visibles en dessous de $-10{ }^{\circ} \mathrm{C}$ (Fig. 7).

Une anomalie de propriété électrique est effectivement obtenue (Fig. 11) pour les basses teneurs en phase intergranulaire vitreuse $\left(3 \%\right.$ recuit à $950{ }^{\circ} \mathrm{C}$, $5 \%$ recuit à $800^{\circ} \mathrm{C}$ ). Une étude plus fine (meilleure résolution expérimentale, teneur de verre variable entre $0.1 \%$ et $10 \%$ ) serait nécessaire pour expliciter le phénomène.

\section{Remerciements.}

S. Chorlet est remercié pour sa contribution à la synthèse des composites et à la mesure de certaines propriétés électriques.

\section{Bibliographie}

[1] De nombreuses références peuvent être trouvées in Solid State Ionics Conferences Proceedings, e.g. SSI 83, Solid State Ionics 9/10 (1983), SSI 85, Solid State Ionics 18/19 (1986).

[2] Hagenmuller, P. et Van Gool, W., eds, Solid electrolytes (Academic Press, New York) 1978.

[3] Wheat, T. A., Ahmad, A. et Kuriakose, A. K., eds, Progress in Solid Electrolytes (CANMET, Energy, Mines and Resources, Ottawa) 1983.

[4] Gordon, R. S., Miller, C. R., MAC Entire, B. J., BeCK, E. D. et Rasmussen, J. R., Solid State Ionics 3/4 (1981).

[5] Kuriakose, A. K., Wheat, T. A., Ahmad, A. and Dirocco, J., J. Am. Ceram. Soc. 67 (1984) 179.

[6] Colomban, Ph., in: Reactivity of Solids (XI ICORS ; Dijon 27-8, 1-9 1984), Barret P. and Dufour L. C., Dufour, eds. (Elsevier, Amsterdam) 1985 , p. 487.

[7] Colomban, Ph., Solid State Ionics, 21 (1986) 97.

[8] Perthuis, H. et Colomban, Ph., Mater. Res. Bull. 19 (1984) 621.

[9] Perthuis, H. et Colomban, Ph., Ceramics Int. 12 (1986) 39.

[10] Pham-Thi, M., Adet, Ph., Velasco, G. et ColomBAN, Ph., Appl. Phys. Lett. 48 (1986) 1348.

[11] Liang, C. C., Pats U.S. 3, 730, 775 ; 3, 713, 897.

[12] MAIER, J., Solid State Ionics $18 / 19$ (1986) 1141.

[13] Colomban, Ph., in Advances in Ceramics 21 (1987).

[14] Von Alpen, U., Bell, M. F. et Höfer, H. H., Solid State Ionics 3/4 (1981) 215.

[15] Boilot, J. P., Salanié, J. P., Desplanches, G. and Le Potier, D., Mat. Res. Bull. 14 (1979) 1496.
[16] Köhler, H. et Schultz, H., Mat. Res. Bull. 18 (1986) 1143.

[17] BAYARD, M. L. et BARNA, C. G., J. Electroanal. chem. 91 (1987) 201.

[18] Bogusz, W., Phys. Status Solidi (A) 66 (1981) K 109.

[19] Baru, M., Perthuis, H. et Colomban, Ph., Solid State Ionics 11 (1983) 157.

[20] Bouquin, O., Perthuis et Colomban, Ph., J. Mat. Science Lett. 4 (1985) 956.

[21] Bauerle, J. E., J. Phys. Solids, 30 (1969) 2657.

[22] Cole, K. S., Cole, R. H., J. Chem. Phys. 9 (1941) 341.

[23] MaC Donald, J. R., J. Electrochem. Soc. 124 (1987) 1022.

[24] Schouler, E., Giroud, G. et Kleitz, M., J. Chim. Phys. 9 (1973) 1309.

[25] Fabry, P., Schouler, E. J. L. et Kleitz, M., Electrochim. Acta 23 (1978) 539.

[26] Schouler, E. J. L. in Solid State Protonic Conductors III, Goudenough J. B., Jensen J. et Potier A. eds (Odense University Press) 1985, p. 16.

[27] Colomban, Ph., Colloque Int. "Composites Céramique-Céramique », ABVC, Mons, 1315 avril 1987, Silicates Industriels, à paraître.

[28] Thomas, M. G. S. R., Bruce, P. G. et GoodeNOUGH, J. B., J. Electrochem. Soc. 132 (1985) 1521.

[29] Mougenel, J. C. et Kessler, H., in Solid State Protonic Conductors III, Goodenough J. B., Jensen J. et Potier A. eds (Odense University Press) 1985, p. 68.

[30] Perthuis, H., thèse Paris 1983. 